\title{
Skeletal muscle bioenergetics in the chronic fatigue syndrome
}

\author{
Phillip R J Barnes, Doris J Taylor, Graham J Kemp, George K Radda
}

\begin{abstract}
Skeletal muscle bioenergetics and control of intracellular pH have been investigated in 46 patients with chronic fatigue syndrome by phosphorus magnetic resonance spectroscopy. The results have been compared with those from healthy controls and from a group of patients with mitochondrial cytopathies affecting skeletal muscle. No consistent abnormalities of glycolysis, mitochondrial metabolism or pH regulation were identified in the group when taken as a whole, although in 12 of the 46 patients the relationship between pH and phosphocreatine utilisation during exercise fell outside the normal range. Of these, 6 patients showed increased acidification relative to phosphocreatine depletion while 6 showed reduced acidification. These findings do not support the hypothesis that any specific metabolic abnormality underlies fatigue in this syndrome although abnormalities may be present in a minority of patients.
\end{abstract}

(F Neurol Neurosurg Psychiatry 1993;56:679-683)

The chronic fatigue syndrome $(\mathrm{CFS})^{1}$ consists of profound fatigue precipitated by minimal physical exertion and commonly follows an episode clinically resembling viral infection. There is frequently muscle pain which is present at rest and exacerbated by exercise. Non-muscular features include impairment of concentration and memory, depression, headaches, tinnitus and limb paraesthesiae. Despite these well-described symptoms, clinical examination and routine investigations are generally normal ${ }^{2}$ and there remains considerable debate as to whether the syndrome is of organic or psychological origin.

Muscle biopsy studies have given conflicting results. An early study ${ }^{3}$ reported widely scattered single necrotic fibres without inflammation together with type II fibre predominance in a majority of patients. A more recent study ${ }^{4}$ has failed to confirm these findings, however, and in a recent publication the authors of the first paper reported type II fibre atrophy. ${ }^{5}$ In this paper they also reported ultrastructural changes in $80 \%$ of biopsies that they interpreted as mitochondrial degeneration, although there were no quantitative data on the percentages of mitochondria affected other than that the findings were more "severe" and less "rare" than those in the $52 \%$ of control biopsies which also showed some mitochondrial change. The authors proposed that an acquired mitochondrial defect might account for symptoms of fatigue and myalgia. Nevertheless, in vitro tests of glycolytic and mitochondrial respiratory chain enzyme activities have either shown normal activities or non-specific global reductions in mitochondrial activity. ${ }^{67}$ Our group has previously reported results from a single patient with chronic fatigue syndrome who showed excessive intracellular acidification during exercise when studied using in vivo ${ }^{31} \mathrm{P}$ magnetic resonance spectroscopy (MRS). ${ }^{8}$ Later data from a selected group of 6 patients were presented in whom 5 showed similar acidification, although this was disproportionate to phosphocreatine utilisation in only two (which included the Lancet case). ${ }^{9}$ The latter data were reviewed by Yonge $^{10}$ who also showed that the abnormalities observed were seen in other conditions as well. We present a detailed analysis of the findings on 46 patients with chronic fatigue syndrome, and discuss the results in relation to our current understanding of the bioenergetics of skeletal muscle.

\section{Methods and subjects}

Phosphorus magnetic resonance spectroscopy

Spectra were collected from the forearm muscle flexor digitorum superficialis using techniques as previously described. ${ }^{11}$ Briefly, the subject lay supine with the arm abducted to $90^{\circ}$ and placed so that the flexor digitorum superficialis lay in the centre of a 1.89 Tesla $30 \mathrm{~cm}$ bore superconducting magnet which was interfaced with a Fourier transform spectrometer (Oxford Research Systems, Oxford, UK). Radiofrequency pulses of $20 \mu$ s length were delivered at $2 \mathrm{~s}$ intervals using a $2.5 \mathrm{~cm}$ diameter surface coil, thus optimising ${ }^{31} \mathrm{P}$ signal collection. The $90^{\circ}$ pulse with this coil was $16 \mu \mathrm{s}$. A spectrum consisting of 128 accumulated free induction decays (FIDs) was collected from resting muscle to provide a baseline for exercise and recovery. During exercise (see below) spectra were collected over $64 \mathrm{~s}$ (32 FIDs) and during recovery they were collected over 32 or $64 \mathrm{~s}$ for a period of 11 minutes.

Determination of intracellular metabolite concentrations and $\mathrm{pH}_{i}$

Metabolite ratios were calculated from the peak areas of phosphocreatine (PCr), inorganic phosphate $\left(\mathrm{P}_{\mathrm{i}}\right)$, and $\beta$-ATP (cor- 
Table 1 Metabolite ratios in resting skeletal muscle

\begin{tabular}{|c|c|c|c|c|c|}
\hline \multirow{8}{*}{$\begin{array}{l}\text { Group } \\
\text { n } \\
\mathrm{pH}_{\mathrm{i}} \\
\text { PCr/ATP } / \mathrm{ATP} \\
\mathrm{P}_{\mathrm{i}} / \mathrm{ATP} \\
\mathrm{PCr} \mathrm{P}_{\mathrm{i}} \\
\mathrm{PCr} /\left(\mathrm{PCr}+\mathrm{P}_{\mathrm{i}}\right) \\
\text { ADP }(\mu \mathrm{M})\end{array}$} & \multirow{2}{*}{$\begin{array}{l}\text { Controls } \\
\text { A } \\
19\end{array}$} & \multirow{2}{*}{$\frac{P^{*}}{(A \text { vs } B)}$} & \multicolumn{2}{|c|}{ Mito Myopathy $P^{*}$} & \multirow{2}{*}{$\begin{array}{l}\text { CFS } \\
\mathrm{C} \\
46\end{array}$} \\
\hline & & & $\begin{array}{l}\text { B } \\
21\end{array}$ & (B vs $\mathrm{C})$ & \\
\hline & $7.03(0.03)$ & 0.67 & $7.03(0.05)$ & 0.25 & $7.03(0.03)$ \\
\hline & $3.08(0.19)$ & 0.0001 & $2.52(0.40)$ & 0.0001 & $3.08(0.08)$ \\
\hline & $0.34(0.07)$ & 0.05 & $0.40(0.11)$ & 0.08 & $0.34(0.1)$ \\
\hline & $9.40(1.90)$ & 0.002 & $6.90(3.20)$ & 0.0002 & $9.66(3.39)$ \\
\hline & $0.90(0.02)$ & 0.001 & $0.86(0.05)$ & 0.0003 & $0.9(0.03)$ \\
\hline & $6(3)$ & 0.0001 & $22(13)$ & 0.0001 & $6(5)$ \\
\hline
\end{tabular}

All values given as means with standard deviations in parentheses.

$\star P$ values refer to differences between the groups stated at the head of the column in which they appear. There was no significant difference between patients with CFS and controls for any parameter $(p>0.5$ for each).

rected for differential saturation effects)..$^{12}$ Intracellular $\mathrm{pH}\left(\mathrm{pH}_{\mathrm{i}}\right)$ was calculated from the chemical shift difference of the $P_{i}$ peak relative to the $\mathrm{PCr}$ peak as previously described. ${ }^{12}$

Free cytosolic ADP concentrations were calculated from the creatine kinase equilibrium expression: ${ }^{13}$

$$
\mathrm{K}_{\mathrm{eq}}=\frac{[\mathrm{ATP}][\text { creatine }]}{[\mathrm{ADP}]\left[\mathrm{H}^{+}\right][\mathrm{PCr}]}
$$

taking $\mathrm{K}_{\mathrm{eq}}$ as $1.66 \times 10^{9}$ and assuming total creatine (PCr + creatine) of $42.5 \mathrm{mM}$ and total ATP of $8.2 \mathrm{mM} .^{11}$ The concentration of $\mathrm{PCr}$ during exercise is conveniently expressed as the ratio $\mathrm{PCr} /\left(\mathbf{P C r}+\mathbf{P}_{\mathrm{i}}\right)$, since $\left(\mathrm{PCr}+\mathrm{P}_{\mathrm{i}}\right)$ remains constant during exercise and this ratio corrects for changes in absolute signal intensity due to movement of the muscle relative to the coil.

Recovery half-times were calculated from the semilog transformation of the first four data points in recovery and initial rates of $\mathrm{PCr}$ recovery (which are a direct measure of mitochondrial ATP synthesis) ${ }^{14}$ from the calculated $\mathrm{PCr}$ concentration at the mid-point of the first data point in recovery. Where the utilisation of $\mathrm{PCr}$ during exercise was less than $30 \%$ the recovery data were excluded from the group analysis since estimates of
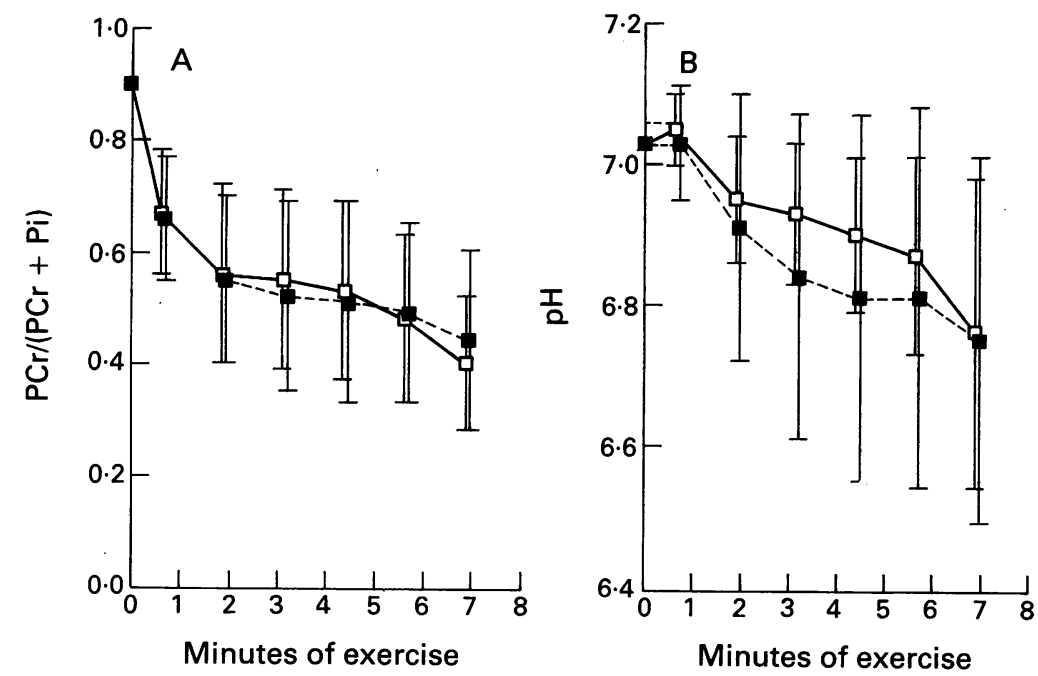

Figure 1 (A) Changes in the ratio $P C r /(P C r+P)$ during exercise in patients with $C F S$ (dotted line and closed symbols) and controls (solid line and open symbols). Means (1 SD); (B) Changes in $\mathrm{pH}_{i}$ during the same exercise protocol (symbols as for fig $1 \mathrm{~A}$ ). recovery times are unreliable in these circumstances. One out of the 19 control subjects and 6 out of 46 patients with CFS satisfied this criterion and their recovery data are not included.

\section{Exercise protocol}

All controls and patients performed an exercise protocol which consisted of squeezing a rubber sphygmomanometer bulb 22 times per minute. During the first 5 minutes of exercise the maximum pressure that could be generated by emptying the bulb was preset at $100 \mathrm{~mm} \mathrm{Hg}$ and this was increased to $300 \mathrm{~mm}$ $\mathrm{Hg}$ for the final 2.5 minutes. Subjects who could not achieve these pressures simply squeezed as hard as they could.

\section{Statistical analysis}

All data are expressed as their mean with standard deviation (SD) in parentheses unless otherwise stated, and a range is given for some control parameters. Significance was calculated using the Mann-Whitney U test.

\section{Subjects}

The data presented are from 46 patients (24 men and 22 women aged 18-69 years; mean 36) referred by hospital specialists with a diagnosis of a chronic fatigue syndrome, and include the cases previously reported by us. ${ }^{89}$ In all cases there was a clear clinical history of a preceding viral-type illness followed by incapacitating fatigue and in no case was there evidence of any other illness to account for the fatigue or other symptoms. Symptoms had been present for a mean of 35 months (range 6-132) at the time of study and all cases satisfied the Green College criteria for diagnosis of a chronic fatigue syndrome. ${ }^{1}$ In 21 cases there was additional laboratory evidence of recent viral infection at the time of referral (thus satisfying the strict criteria for diagnosis of a post-infectious fatigue syndrome) ${ }^{1}$; in the remaining 25 patients tests had either not been performed or they were negative. Data are also shown from 21 patients with biopsy-proven mitochondrial cytopathies (ages 12-70 years; mean 33). Control subjects were sedentary volunteers (ages 20-79; mean 41) and do not include any trained athletes. All studies were approved by the Central Oxford Research Ethics Committee and informed verbal and written consent was obtained in all cases.

\section{Results}

Resting muscle

Results for resting skeletal muscle are shown in table 1. There were no significant differences between patients with chronic fatigue syndrome and controls. Patients with mitochondrial myopathies had significantly lower $\mathrm{PCr} / \mathrm{ATP}$ and $\mathrm{PCr} / \mathrm{P}_{\mathrm{i}}$ ratios and higher resting [ADP] compared with either controls or patients with CFS. Subgroup analysis of those patients with CFS in whom positive evidence was found of viral infection showed no difference in resting muscle compared 
Table 2 Recovery of metabolites after exercise

\begin{tabular}{llllll}
\hline & Controls & $P^{*}$ & $\begin{array}{l}\text { Mitochondrial } \\
\text { myopathy }\end{array}$ & $P^{*}$ & CFS \\
\hline Group & $\mathrm{A}$ & (A vs B) & B & (B vs C) & C \\
Init rate PCr recovery (mM/min) & $20(13)$ & 0.10 & $13(8)$ & 0.007 & $20(9)$ \\
$\mathrm{t}_{1 / 2} \mathrm{PCr}(\mathrm{s})$ & $50(17)$ & 0.04 & $92(62)$ & 0.02 & $53(30)$ \\
$\mathrm{t}_{1 / 2} \mathrm{P}_{\mathrm{i}}(\mathrm{s})$ & $28(7)$ & 0.004 & $65(44)$ & 0.003 & $31(11)$ \\
$\mathrm{t}_{1 / 2} \mathrm{ADP}(\mathrm{s})$ & $14(7)$ & 0.0004 & $53(68)$ & 0.0001 & $14(5)$ \\
\hline
\end{tabular}

All values given as means with standard deviations in parentheses.

${ }^{\star} P$ values refer to differences between the groups stated at the head of the column in which they appear. There was no significant difference between patients with CFS and controls for any parameter $(p>0.4$ for each)

with controls or those CFS patients without evidence of viral infection (data not shown).

\section{Exercise and recovery}

The changes in $\mathrm{PCr}$ (expressed as $\mathrm{PCr} /$ $\left(\mathrm{PCr}+\mathrm{P}_{\mathrm{i}}\right)$ ) and $\mathrm{pH}_{\mathrm{i}}$ during exercise are summarised in fig 1 . There are no significant differences between the CFS patient group and controls, although the scatter in the $\mathrm{pH}_{\mathrm{i}}$ data from patients was greater. Data from recovery are shown in table 2 . Again there are no differences between the control and CFS groups (including the CFS subgroup with positive evidence of viral infection), whereas patients with mitochondrial myopathy have prolonged recovery half times for $[\mathrm{PCr}],\left[\mathrm{P}_{\mathrm{i}}\right]$ and $[\mathrm{ADP}]$. Recovery data for $\mathrm{pH}$ were only obtainable in a minority of patients and

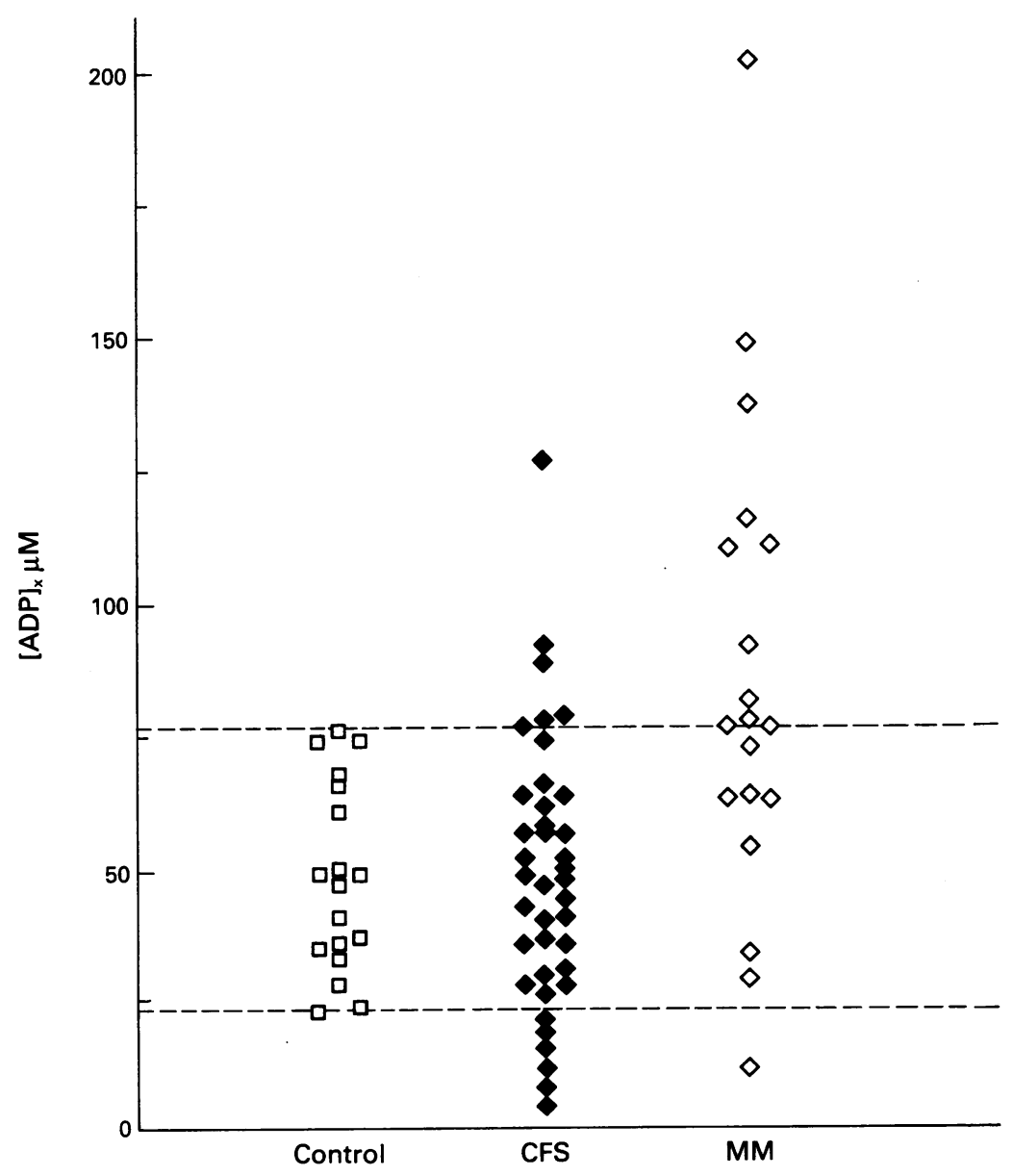

Figure 2 Distribution histogram of the free $[A D P]$ at the end of exercise ([ADP $]_{\searrow}$ in controls, patients with CFS and patients with mitochondrial myopathies (MM). The dotted lines show the upper and lower limits of the control range. controls due to loss of the $P_{i}$ signal during recovery ${ }^{12}$ and therefore are not presented here.

The relationship between changes in [PCr] and $\mathrm{pH}_{\mathrm{i}}$ during exercise may be expressed as the calculated free [ADP], since this is derived from the creatine kinase equilibrium expression in which [PCr] and $\mathrm{pH}_{\mathrm{i}}$ are the only other major variables. When $\mathrm{pH}_{\mathrm{i}}$ is lower for a given [PCr] than that in controls, the calculated [ADP] is lower than in controls. Figure 2 shows the [ADP] at the end of exercise $\left([\mathrm{ADP}]_{\mathrm{x}}\right)$ for the three groups of subjects, and the majority of CFS patients fall within the control range. It is possible, however, to identify 6 patients who have lower than normal $[\mathrm{ADP}]_{\mathrm{x}}$, due to greater than normal acidification during exercise. Analysing these separately, there were no significant differences between this subgroup and the control group at rest but the half time for recovery of [PCr] was significantly prolonged (63s [SD 33 ] vs 50 s [17]; $p=0.002$ ). There were also 6 patients with CFS who show an $[\mathrm{ADP}]_{\mathrm{x}}$ above the control range (signifying less acidification of muscle than is usual for the degree of PCr depletion). When compared with controls this subgroup had a lower ratio for PCr/ATP in resting muscle $(2.79$ [SD 0.21 ] vs $3.08[0.19] ; p=0.002)$ and higher [ADP] at rest $(11 \mu \mathrm{M}$ [SD 3] vs $6 \mu \mathrm{M}$ [3]; $\mathrm{p}=$ $0 \cdot 007$ ). Recovery half times were not significantly different from controls, however. If this subgroup is also compared with those patients with mitochondrial myopathy whose $[A D P]_{x}$ is above the normal range (11/21 patients) the latter have a still lower resting PCr/ATP (2.42 [SD 0.48]; p = 0.03) and higher resting [ADP] $(25 \mu \mathrm{M}$ [SD 15]; $p=0.02$ ) and the recovery half time for [ADP] is significantly longer in the mitochondrial myopathy group (65s [SD 79] vs $12 \mathrm{~s}$ [2] in CFS; $p=0.006$ ). Three of these 6 CFS patients with high $[\mathrm{ADP}]_{\mathrm{x}}$ had had muscle biopsies and all were normal without evidence of ragged red fibres.

\section{Discussion}

The pathophysiological basis of the chronic fatigue syndrome (including post-viral fatigue syndrome, "myalgic encephalomyelitis" and neurasthenia) remains a mystery. Many of the symptoms relate to muscle with prominent fatigue and pain at rest and on minimal exertion. Exertional muscle pain is also a feature of a number of metabolic myopathies including glycogenoses of muscle, fatty acid oxidation defects and adenylate deaminase deficiency although it is uncommon in mitochondrial myopathies. A previous publication from our group ${ }^{8}$ reported results from a single patient with post-varicella fatigue syndrome who showed a repeatable abnormal metabolic response to aerobic exercise with greater acidification than that seen in controls and slow recovery of [PCr] following exercise. This was interpreted as being due to abnormal control of glycolysis leading to increased lactic acid production, although others have 
used the data as evidence of mitochondrial dysfunction. ${ }^{5}$ We have shown here that in a group of 46 patients with CFS following a standard exercise protocol there are no consistent bioenergetic abnormalities. In particular in the group as a whole there is no evidence of a mitochondrial defect nor of a consistent abnormality of $\mathrm{pH}_{\mathrm{i}}$ regulation. This is in keeping with other in vitro studies of mitochondrial and glycolytic function ${ }^{67}$ and with preliminary results using ${ }^{31} \mathrm{P}$ MRS to study the tibialis anterior. ${ }^{15}$ Nevertheless, the variation in the results from CFS patients was greater than that seen in controls and, since there are difficulties in the diagnosis of CFS and the possibility exists that it represents the clinical manifestations of a number of as yet unidentified pathologies, it is pertinent to consider further the metabolic responses of those subgroups of patients described above who fall outside the normal range.

Because the degree of metabolic change in muscle during exercise will depend to a large degree on the level of fitness and "training" it is most appropriate to compare metabolic parameters rather than to look at each separately. There is known to be a well defined relationship between changes in $\mathrm{pH}_{\mathrm{i}}$ and the utilisation of $\mathrm{PCr} .^{12}$ In particular, significant acidification of muscle does not occur until $\mathrm{PCr}$ falls to approximately $60 \%$ of its resting level. In those patients with CFS who acidify more than controls and whose $[\mathrm{ADP}]_{\mathrm{x}}$ therefore is low (fig 2), this "threshold" for acidification is unchanged (data not shown). This pattern of $\mathrm{pH}_{\mathrm{i}}$ response is also quite different from that in patients with mitochondrial myopathies $^{11}$ (see results). In the latter the degree of intracellular acidification is less than in controls despite the lactic acidaemia which is often present ${ }^{16}$ and possibly represents a compensatory increase in proton extrusion from the cell. The group of CFS patients with marked acidification do have slow [PCr] recovery but this is to be expected since their $[\mathrm{ADP}]_{\mathrm{x}}$ is low and rates of mitochondrial ATP synthesis (and thus PCr resynthesis) ${ }^{17}$ depend upon [ADP] in vivo. ${ }^{14}$ Further evidence against a significant mitochondrial defect in this subgroup is the normal recovery half time for [ADP] which is itself a sensitive indicator of mitochondrial function. ${ }^{17}$ The response to exercise in this subgroup resembles that seen in a group of healthy volunteers exercising at a much higher than normal workload (D Taylor, G Kemp, unpublished data) in which the $\mathrm{pH}_{\mathrm{i}}$ fell rapidly and $[A D P]_{x}$ was low. In the high workload control group the recovery half time for [PCr] was low (as expected for the low $[\mathrm{ADP}]_{\mathrm{x}}$ ) but that for $[\mathrm{ADP}]$ itself was normal. This shows that changes in relative work done affect the relative aerobic and anaerobic contributions to ATP synthesis and suggest that different degrees of fitness may explain the results. In particular, studies of trained athletes show that they have improved oxidative metabolism and reduced acidification during exercise both in specifically trained and other muscle groups. ${ }^{1819}$ "Detraining" due to reduced activity is likely to produce the reverse changes and would explain the present results in this small subgroup.

A similar consideration of that subgroup of CFS patients who have high $[\mathrm{ADP}]_{\mathrm{x}}$ (reflecting less intracellular acidification relative to PCr utilisation than controls) shows that they have slightly lower resting PCr/ATP and higher $[A D P]$ than controls, although they remain significantly different from the mitochondrial myopathy group. Recovery data from this group of CFS patients are entirely normal, however, so that there is no evidence of significant mitochondrial dysfunction. The resting abnormalities may reflect the non-specific mild reductions in mitochondrial enzyme activities reported by others $^{7}$ but they show no evidence of any specific metabolic defect.

In conclusion we have failed to demonstrate any specific metabolic abnormalities in the skeletal muscle of patients with chronic fatigue syndrome. In particular there is no evidence of a functional bioenergetic correlate of the morphological abnormalities reported by Behan $e t a \bar{P}$ in $80 \%$ of their patients. We have found a relationship between intracellular acidification and PCr utilisation that lies outside the control range in 12 out of 46 patients, but equal numbers showed abnormally great and abnormally little acidification. We cannot rule out minor metabolic abnormalities in some of these patients but it is clear that there is no specific bioenergetic abnormality in CFS that can explain the prominent symptoms of fatigue. Furthermore, although some minor electrophysiological abnormalities have been reported on single fibre $\mathrm{EMG}^{20}$ and in protein synthetic rates, ${ }^{21}$ careful assessment of strength and fatigability have shown no evidence for a peripheral neuromuscular component to the symptoms found in the disease and a central cause for fatigue seems more likely. ${ }^{22} 23$

These experiments were carried out over a number of years and many members of the MRC Biochemical and Clinica Magnetic Resonance Unit have contributed to them and their contributions are most gratefully acknowledged. In particular Drs D Arnold, P Bore, B Rajagopalan and P Styles were closely involved in the planning of studies and in early experiments. The assistance of Mrs R Cooper in collating the data is also acknowledged. We thank those clinicians who have referred their patients to us. PRIB and GJK acknowled have referred their patients to us. PRJB and GJK acknowledge personal support from the Muscular Dystrophy Group of Great Britain and Northern Ireland. The Unit is supported by the
Medical Research Council and the British Heart Foundation.

1 Sharpe MC, Archard LC, Banatvala JE, et al. A reportchronic fatigue syndrome: guidelines for research. $\mathcal{F} R$ Soc Med 1991;84:118-21.

2 Behan PO, Bakheit AMO. Clinical spectrum of postviral fatigue syndrome. Br Med Bull 1991;47:793-808.

3 Behan PO, Behan WMH, Bell EJ. The postviral fatigue syndrome-an analysis of the findings in 50 cases. $f$ Infect 1985;10:211-22.

4 Teahon K, Preedy VR, Smith DG, Peters TJ. Clinical studies of the postviral fatigue syndrome (PVFS) with special reference to skeletal muscle function. Clin $\mathrm{Sc}$ 1988;75:45

5 Behan WMH, More IAR, Behan PO. Mitochondrial abnormalities in the postviral fatigue syndrome. Acta Neuropathol (Berl) 1991;83:61-5.

6 Byrne E, Trounce I. Chronic fatigue and myalgia syndrome: mitochondrial and glycolytic studies in skeletal muscle. $\mathcal{F}$ Neurol Neurosurg Psychiatry 1987;50:743-6.

7 Wagenmakers AJM, Kaur N, Coakley JH, Griffiths RD, Edwards RHT. Mitochondrial metabolism in myopathy and myalgia. In: Benzi G, ed. Advances in myochemistry. London: Fohn Libby Eurotext, 1987:219-30. 
8 Arnold DL, Bore PJ, Radda GK, Styles P, Taylor DJ. Excessive intracellular acidosis of skeletal muscle on excercise in a patient with a post-viral exhaustion/fatigue syndrome. A ${ }^{31} \mathrm{P}$ nuclear magnetic resonance study. syndrome. A ${ }^{31} \mathrm{P}$ nucle

9 Arnold DL, Bore PJ, Radda GK, Styles P, Taylor DJ. Excessive intracellular acidosis of skeletal muscle on exercise in the post-viral exhaustion/fatigue syndrome: a ${ }^{31}$ P NMR study. Proc 3rd Annual meeting of the Society for Magnetic Resonance in Medicine, New York. 1984:12-13

10 Yonge RP. Magnetic resonance muscle studies: implications for psychiatry. $7 R$ Soc Med 1988;81:322-6.

11 Arnold DL, Taylor DJ, Radda GK. Investigation of human mitochondrial myopathies by phosphorus magnetic resonance spectroscopy. Ann Neurol 1985;18: 189-96.

12 Taylor DJ, Bore PJ, Styles P, Gadian D, Radda GK Bioenergetics of intact human muscle. A ${ }^{31} \mathrm{P}$ nuclear Bioenergetics of intact human muscle. A $31 \mathrm{P}$ nuclear

13 Veech RL, Lawson JWR, Cornell NW, Krebs HA Cytosolic phosphorylation potential. I Biol Chem 1979; 254:6538-47.

14 Taylor DJ. Control of mitochondrial energy production in vivo. In: Azzi A, Nalecz KA, Wotczak L, eds. Anion carriers of mitochondrial membranes. Berlin: Springer-Verlag 1989;373-81.

15 Kent-Braun J, Sharma K, Chien C-C, Weiner M, Miller RG. Chronic fatioue syndrome: pathophysiologic basis for muscular fatigue. Neurology 1991;41(suppl 1):395.
16 Petty RWH, Harding AE, Morgan-Hughes JA. The clinical features of mitochondrial myopathy. Brain cal features of

17 Arnold DL Matthews PM, Radda GK. Metabolic recovery after exercise and the assessment of mitochondrial function in vivo in human skeletal muscle by means of 'P NMR. Magn Reson Med 1984;1:307-15.

18 Park JH, Brown RL, Park CR, Cohn M, Chance B. Energy metabolism of the untrained muscle of elite runners as observed by ${ }^{31} \mathrm{P}$ magnetic resonance spectroscopy: Evidence suggesting a genetic endowment for endurance exercise. Proc Natl Acad Sci USA 1988; 85:8780-4.

19 McCully KK, Boden BP, Tuchler M, Fountain MR Chance B. Wrist flexor muscles of elite rowers measured with magnetic resonance spectroscopy. If Appl Physiol 1989;67:926-32.

20 Jamal GA, Hansen S. Electrophysiological studies in the post-viral fatigue syndrome. $\mathcal{f}$ Neurol Neurosurg Psychiatry 1985;48:691 4.

21 Edwards RHT, Newham DJ, Peters TJ. Muscle pathology and biochemistry. Br Med Bull 1991;47:826-37.

22 Stokes MJ, Cooper RG, Edwards RHT. Normal muscle strength and fatigability in patients with effort syndromes. BMF 1988;297:1014-7.

23 Lloyd AR, Hales JP, Gandevia SC. Muscle strength, endurance and recovery in the post-infection fatigue syndrome. I Neurol Neurosurg Psychiatry 1988;51: 1316-22. 